\title{
POTENCIAL EVOCADO AUDITIVO DE ESTADO ESTÁVEL COM ESTÍMULO DE RUÍDO BRANCO MODULADO EM AMPLITUDE EM TRIAGEM AUDITIVA NEONATAL
}

\section{Neonatal hearing screening using auditory steady state responses with amplitude modulated white noise stimuli}

\author{
Danielle Gomes Pinto ${ }^{(1)}$, Silvana Maria Sobral Griz ${ }^{(2)}$, Otávio Gomes Lins ${ }^{(3)}$
}

\begin{abstract}
RESUMO
Objetivo: verificar a utilização do estímulo ruído branco, modulado em amplitude, no exame de Potencial Evocado Auditivo de Estado Estável (PEAEE). Método: foram avaliados 30 neonatos e lactentes, na Maternidade do Hospital das Clínicas da UFPE, que realizaram os exames de Emissões Otoacústicas Transientes (EOAT) e PEAEE, com ruído branco de amplitude modulada. Resultados: para o exame de PEAEE, sugere-se que a intensidade ideal para o ponto de corte seja $50 \mathrm{~dB}$ NPS, para os casos com passa na triagem auditiva, com o exame de EOAT. Conclusão: o exame de PEAEE, realizado com ruído branco, modulado em amplitude, parece ser promissor como ferramenta de triagem auditiva. Entretanto, estudos são necessários para observar a relação entre os limiares das respostas auditivas de estado estável utilizando-se tons puros e ruído branco, a fim de melhor elucidar e definir parâmetros e protocolos de sua utilização.
\end{abstract}

DESCRITORES: Triagem; Audição; Potenciais Evocados Auditivos

\section{INTRODUÇÃO}

Segundo o Comitê Brasileiro sobre Perdas Auditivas na Infância'1 (2001), a incidência de surdez é de aproximadamente 1 a 3 recém-nascidos em cada mil nascimentos e aumenta para $1 \%$ a $5 \% \mathrm{em}$ bebês provenientes de unidades de terapia intensiva neonatal. Em mil crianças nascidas no Brasil, de duas a sete apresentam problemas de surdez.

O Joint Comitte on Infant Hearing ${ }^{2}$ (2000) recomenda que todas as crianças com perda auditiva

(1) Fonoaudióloga; Professora substituta do Departamento de Fonoaudiologia da Universidade Federal de Pernambuco, UFPE, Recife, Pernambuco, Brasil; Mestre em Neuropsiquiatria e Ciências do Comportamento pela Universidade Federal de Pernambuco.

(2) Fonoaudióloga; Professora Adjunta do Departamento de Fonoaudiologia da Universidade Federal de Pernambuco UFPE, Recife, Pernambuco, Brasil; Doutora em Psicologia Cognitiva pela Universidade Federal de Pernambuco.

(3) Médico; Professor Adjunto do Departamento de Medicina da Universidade Federal de Pernambuco - UFPE, Recife, Pernambuco, Brasil; Doutor em Neurologia pela Universidade de São Paulo.

Conflito de interesses: inexistente congênita ou neonatal devem ser identificadas antes dos 3 meses de idade e que o processo de intervenção inicie antes dos 6 meses de idade. Os procedimentos de triagem auditiva neonatal devem envolver exames eletrofisiológicos, tais como o exame do Potencial Evocado Auditivo de Tronco Encefálico (PEATE) e o exame de Emissão Otoacústica Evocada (EOAE).

Por se tratar de um exame eletrofisiológico, capaz de avaliar objetivamente as respostas da via auditiva, de maneira rápida, surgiu a possibilidade do uso do exame de potencial evocado auditivo de estado estável (PEAEE), por meio da análise das respostas auditivas de estado estável (RAEE). Estas respostas são ondas contínuas periódicas eliciadas por tons contínuos ou por estímulo do tipo ruído branco, que podem ser de amplitude ou freqüência moduladas $^{3}$. O exame de PEAEE tem sido utilizado para fins de diagnóstico audiológico e triagem auditiva. Entretanto, seu uso em triagem ainda não está estabelecido. Alguns autores sugerem que o ruído branco pode ser o estímulo escolhido para captação das RAEE. Entretanto, não há, até então, estudos 
clínicos sobre a sua aplicação, apenas a sugestão de sua utilização ${ }^{3-5}$.

O presente estudo teve como objetivo geral verificar a utilização do estímulo ruído branco, modulado em amplitude, no exame de PEAEE, para que, no futuro, este possa ser utilizado como ferramenta de triagem auditiva neonatal. Para isso, foram analisados: (1) aspectos relativos às características da população; (2) os resultados dos exames de EOAT e PEAEE, (3) a correlação entre os resultados dos exames de EOAT e PEAEE, e (4) o tempo de realização do exame de RAEE, considerando-se o número de amostras obtidas.

\section{MÉTODO}

Participaram do estudo 30 neonatos e lactentes atendidos no programa de triagem auditiva neonatal, do Hospital das Clínicas da UFPE (HC-UFPE). O exame de EOAT foi realizado com o equipamento marca Madsen, modelo Capella. Utilizou-se o programa Quickscreen, com modo não-linear de apresentação do clique, com duração de $80 \mathrm{~ms}$ e faixa de freqüência de $400 \mathrm{a} 6000 \mathrm{~Hz}$. A intensidade do estímulo não ultrapassou $80 \mathrm{~dB}$ peNPS. Foi considerado'passa' o exame de EOAT que consistiu em: reprodutibilidade em pelo menos três bandas consecutivas de $70 \%$ e relação sinal/ ruído igual ou superior a $6 \mathrm{~dB}$, em pelo menos três bandas consecutivas, em ambas as orelhas.

$O$ exame de PEAEE foi realizado com um sistema de aquisição e análise (MASTER - Multiple Auditory Steady-State Response), desenvolvido no Laboratório do Dr. Terry Picton, Rotman Research Institute, Baycrest Centre, North Yook, Canadá6-8. Para preparação da pele, utilizou-se álcool a $70 \%$ e esfoliação com pasta abrasiva. A colocação dos eletrodos se deu nas seguintes posições: eletrodo ativo anterior na posição $\mathrm{Fz}$, eletrodo de referência, no mastóide direito (M2) e eletrodo terra, no mastóide esquerdo (M1). O estímulo utilizado foi ruído branco, modulado em amplitude (modulação exponencial - $A M^{2}$ ) nas freqüências de $84,96 \mathrm{~Hz}$, para a orelha direita, e $94,72 \mathrm{~Hz}$, para a orelha esquerda, apresentados simultaneamente. Os estímulos foram apresentados até as respostas, em ambas as orelhas, serem consideradas estatisticamente significantes ou até atingirem o máximo de 48 varreduras (cerca de 12 minutos). Os estímulos foram apresentados seqüencialmente nas intensidades de 40, 50, 60 e 70 dB NPS.

Foi considerada presença de resposta a identificação estatística do pico espectral, na freqüência da modulação do estímulo portador (ruído branco). A amplitude desta freqüência foi estaticamente superior a amplitude do ruído de fundo, no gráfico do FFT.
A significância foi calculada utilizando-se os testes estatísticos $\mathrm{T}^{2}$ e $\mathrm{F}$ (Lins et al,1995), realizados pelo próprio programa MASTER. $O$ valor de $p$ utilizado foi de 0.05. As RAEE foram consideradas presentes quando os testes estatísticos identificavam uma amplitude da resposta, na freqüência de modulação do ruído branco, em cada orelha, superior ao ruído, até atingir o máximo de 48 varreduras.

O projeto foi aprovado pelo Comitê de Ética em Pesquisa, do Centro de Ciências da Saúde da Universidade Federal de Pernambuco, sob o número de protocolo 256/08. Todas as mães que aceitaram participar da pesquisa assinaram um Termo de Consentimento Livre e Esclarecido (TCLE).

A análise dos dados foi realizada utilizandose técnicas de estatística descritiva e inferencial, utilizando-se o programa Microsoft Excel 2002. A estatística descritiva envolveu a obtenção de distribuições absolutas e percentuais, média, desvio padrão e coeficiente de variação, para caracterização da população, em relação às variáveis idade e presença de indicador de risco para perda auditiva. A estatística inferencial envolveu o cálculo da correlação entre os achados de 'passa' e 'falha' dos exames de EOAT e a presença e ausência de RAEE, por meio da construção de uma tabela de contingência e para avaliar a significância, foram utilizados o teste exato de Fisher, considerando-se um intervalo de confiança de $95 \%$ e o coeficiente Kappa para observar a concordância entre os achados.

A partir da observação da concordância entre as técnicas, pôde-se sugerir uma intensidade como ponto de corte 'passa'/'falha', a ser utilizada na triagem auditiva em neonatos e lactentes.

\section{RESULTADOS}

Os resultados serão apresentados de forma a: (1) caracterizar a população do estudo, (2) apresentar os resultados dos exames de EOAT e de PEAEE, (3) apresentar os resultados da concordância entre o 'passa'/'falha' para o exame de EOAT e presença de respostas/ausência de RAEE, e (4) analisar o tempo de realização do exame de PEAEE, considerando-se o número de amostras obtidas, para se verificar a presença de resposta, por meio da análise da significância realizada pelo MASTER.

Foram avaliados 30 neonatos e lactentes, com idade média de 27, 4 dias e desvio padrão de 58,5 dias. Foram observados indicadores de risco para perda auditiva, descritos pelo $\mathrm{JCIH}$ (2007), em $50 \%(n=15)$ da população, distribuídos da seguinte forma: (a) história familiar de perda auditiva 
$(13,4 \%, n=4)$, (b) permanência em unidade de tratamento intensivo, por um período superior a 5 dias $(16,7 \%, n=5)$ e, (c) uso de ventilação mecânica por um período superior a 5 dias $(20 \%, n=6)$. Houve também uma alta freqüência de baixo peso $(33,5 \%$, $\mathrm{n}=10)$ e prematuridade $(30 \%, \mathrm{n}=9)$, que por si só não se caracterizam como indicadores de risco, mas podem estar relacionados aos indicadores atualmente descritos.

Os resultados dos exames de EOAT foram analisados segundo os índices de 'passa' e 'falha'. Observou-se que houve um alto índice de 'falha' $(10 \%, n=3)(\mathrm{JClH}, 2007)$. Foi remarcado o retorno para a determinação do tipo e grau de perda entre as crianças que falharam, por meio da realização do exame de PEATE (Potencial Evocado Auditivo de Tronco Encefálico) e imitânciometria acústica, bem como o devido encaminhamento para realização de avaliação otorrinolaringológica, e reabilitação, porém não houve retorno das mesmas, o que se deveu provavelmente porque os responsáveis apresentavam baixa renda, o que pode ter dificultado o acesso a transportes, e baixo nível de instrução para o ideal entendimento da necessidade indispensável de diagnóstico e intervenção.

Quanto aos resultados dos exames de PEAEE, para a intensidade de $40 \mathrm{~dB}$ NPS foram observadas presenças de respostas em $83,3 \%(n=25)$,na intensidade de $50 \mathrm{~dB}$ NPS houve resposta em $90 \%$ $(\mathrm{n}=27)$, na intensidade de $60 \mathrm{~dB}$ NPS , respostas em $93,3 \%(n=28)$ e na intensidade de $70 \mathrm{~dB}$ NPS em $100 \%(n=30)$. Observa-se que à medida que aumentou a intensidade, aumentou o índice de presença de PEAEE. Entretanto, apenas a intensidade de $50 \mathrm{~dB}$ NPS confirmou os resultados do exame de EOAT (Tabela 1).

Tabela 1 - Relação entre 'passa' e 'falha' no exame de EOAT e presença e ausência de respostas no RAEE, para as intensidades testadas, por participantes

\begin{tabular}{|c|c|c|c|c|c|c|c|}
\hline $\begin{array}{l}\text { Intensidade } \\
\text { do estímulo }\end{array}$ & $\begin{array}{c}\text { 'falha' EOAT e } \\
\text { ausência de } \\
\text { respostas RAEE }\end{array}$ & $\begin{array}{l}\text { 'passa' EOAT' } \\
\text { e presença } \\
\text { de respostas } \\
\text { no RAEE }\end{array}$ & $\begin{array}{c}\text { 'passa' EOAT } \\
\text { e ausência } \\
\text { de respostas } \\
\text { no RAEE }\end{array}$ & $\begin{array}{c}\text { 'falha' EOAT } \\
\text { e presença } \\
\text { de respostas } \\
\text { no RAEE }\end{array}$ & $\begin{array}{l}\text { Teste } \\
\text { exato de } \\
\text { Fisher }\end{array}$ & Kappa & $\begin{array}{l}\text { TOTAL } \\
(\mathrm{N}=30)\end{array}$ \\
\hline $40 \mathrm{~dB}$ NPS & 3 & 25 & 2 & 0 & $0.002^{*}$ & 0,714 & 30 \\
\hline $50 \mathrm{~dB}$ NPS & 3 & 27 & 0 & 0 & $0.000^{*}$ & $1^{* *}$ & 30 \\
\hline $60 \mathrm{~dB}$ NPS & 2 & 27 & 0 & 1 & $0.007^{*}$ & 0,783 & 30 \\
\hline $70 \mathrm{~dB}$ NPS & 0 & 27 & 0 & 3 & 1.000 & 0 & 30 \\
\hline
\end{tabular}

* Valores estatisticamente signficantes para o teste exato de Fisher $(p<0,05)$

** Concordância perfeita teste Kappa

Observa-se que somente na intensidade de 50 dB NPS houve $100 \%$ de correlação dos resultados entre os exames de EOAT e de PEAEE, para o intervalo de confiança de $95 \%$ e uma concordância perfeita revelada pelo teste Kappa (Tabela 2). Ou seja, os mesmos participantes que obtiveram 'passa' no exame de EOAT obtiveram presença de respostas no exame de PEAEE $(n=27)$ e os mesmos participantes que obtiveram 'falha' no exame de EOAT obtiveram ausência de respostas no exame de PEAEE $(n=3)$.

A partir dos dados descritos na Tabela 2, sugerese que a intensidade de $50 \mathrm{~dB}$ NPS seja considerada a intensidade do ponto de corte para o exame de PEAEE, quando utilizado em triagem auditiva neonatal.

Tabela 2 - Valores de correlação entre 'falha' nas EOAT e ausência de respostas no exame de PEAEE e 'passa' na EOAT e presença de respostas no exame de PEAEE, para as intensidades testadas

\begin{tabular}{ccc}
\hline $\begin{array}{c}\text { Intensidade do estímulo } \\
\text { (dB NPS) }\end{array}$ & Correlação de Passa & Correlação de Falha \\
\hline 40 & 1 & 0.93 \\
50 & 1 & 1 \\
60 & 0.67 & 1 \\
70 & 0 & 1 \\
\hline
\end{tabular}


A análise do tempo de realização do exame de PEAEE, em minutos, foi realizada a partir da multiplicação do número de varreduras por 16 (tempo de cada amostra em segundos) e dividindo-se por 60 (Tabela 3).

Tabela 3 - Média (desvio padrão) do tempo (em minutos) de realização do exame, para cada intensidade do estímulo

\begin{tabular}{cc}
\hline $\begin{array}{c}\text { Intensidade do } \\
\text { estímulo } \\
\text { (dB NPS) }\end{array}$ & $\begin{array}{c}\text { Tempo de realização } \\
\text { do exame } \\
\text { (minutos) }\end{array}$ \\
\hline 40 & $4.4(\mathrm{DP}-4.0)$ \\
50 & $5.0(\mathrm{DP}-4.0)$ \\
60 & $0.5(\mathrm{DP}-0.7)$ \\
70 & $0.4(\mathrm{DP}-0.6)$ \\
\hline
\end{tabular}

DP - Desvio Padrão

Nesta Tabela, observou-se, que o tempo de realização do exame de PEAEE, em cada intensidade foi curto, fazendo com que o exame de PEAEE seja considerado um técnica rápida. Observa-se que à medida que a intensidade aumentou, as respostas do PEAEE apareceram com tempos mais curtos e com menores números de amostras.

\section{DISCUSSÃO}

Para a população estudada, observou-se que $50 \%$ apresentaram indicadores de risco para perda auditiva, sendo os de maior freqüência: história familiar de perda auditiva, permanência em unidade de tratamento intensivo num período superior a 5 dias e uso de ventilação mecânica por um período superior a 5 dias. Além desses indicadores de risco, o baixo peso e prematuridade foram também descritos. Todos esses fatores podem ter justificado o índice de 'falha' de $10 \%(n=3)$ ao exame de EOAT. Resultados semelhantes têm sido descritos na literatura ${ }^{9}$.

O neonato ou lactente prematuro e de baixo peso geralmente apresenta vários fatores que interferem na colocação da sonda e conseqüentemente, nos resultados do exame de EOAT ${ }^{10-13}$, tais como, tamanho do meato acústico externo, sua ressonância, presença de vérnix nas primeiras 48 horas e ruído no meato acústico externo ${ }^{14-18}$, aumentado a possibilidade de falso-positivos ${ }^{19,20}$ e diminuindo sua especificidade.

Entretanto, mesmo considerando a possibilidade de presença de alterações condutivas para os três casos de 'falha' no exame de EOAT, esses resultados foram confirmados pelo exame de PEAEE, que, teoricamente, não é influenciado por tais alterações.

$O$ exame de PEAEE foi realizado nas intensidades de 40, 50, 60 e 70 dB NPS. Para cada intensidade, foi verificada a presença de PEAEE, tendo sido observado que, à medida que a intensidade aumenta, aumenta a presença dessas respostas. Como a análise da presença de PEAEE se baseia na identificação da amplitude do pico espectral na freqüência da modulação, quanto maior a intensidade do estímulo, mais facilmente se deu a identificação da amplitude desta resposta.

Os achados do exame de PEAEE descritos, para população com mesma faixa etária ${ }^{21-23}$ demonstram que alterações na amplitude das respostas podem estar relacionadas ao desenvolvimento maturacional. Lins et $\mathrm{al}^{7}$ (1996) e John et $\mathrm{al}^{4}$ (2004) afirmam que a amplitude das resposta do PEAEE aumenta nas primeiras semanas de vida. Entretanto, não foi encontrado nenhum estudo que tenha descrito alterações da amplitude, relacionadas à mudança na intensidade, e, conseqüentemente, o estabelecimento da intensidade a ser utilizada como ponto de corte, para a identificação da presença ou ausência de resposta, em programas de triagem auditiva neonatal, ainda não foi estabelecida. Somente a determinação da intensidade de corte para o exame do potencial evocado auditivo de tronco encefálico, utilizado como instrumento de triagem auditiva neonatal, tem sido descrita ${ }^{3-5,21,24}$, sem entretanto, relacionar a uma faixa etária específica. Estudos clínicos que utilizam o estímulo ruído branco modulado em amplitude não foram encontrados. Portanto, não há parâmetros definidos para sua testagem.

Os estudos de Picton et $\mathrm{al}^{3}$, John et $\mathrm{al}^{4}$ e Sávio e Perez-Abalo ${ }^{5}$ citam a possibilidade da utilização de estímulos de banda larga, como estímulo, no exame de PEAEE, utilizados na triagem auditiva neonatal. Dessa forma, John et al ${ }^{4}$ destaca a importância da realização de estudos com esta forma de estimulação a fim de se melhor discutir a sua aplicação clínica.

Picton et $\mathrm{al}^{3}$ discutem a utilização de diversos tipos de ruído (banda larga e banda estreita), ao invés da estimulação tonal, mais comumente utilizada nos exames de PEAEE, reforçando, inclusive, que o ruído de banda larga gera maior amplitude de resposta, facilitando sua detecção.

Os resultados desse estudo, utilizando-se o ruído branco modulado em amplitude, como estímulo, no exame do PEAEE, visando sua utilização na triagem auditiva neonatal, sugerem que a única intensidade que confirmou, com $100 \%$ de correlação, os achados do exame de EOAT, foi $50 \mathrm{~dB}$ 
NPS. A relação de proximidade entre presença e ausência de respostas para o exame de PEAEE e passa e falha do exame de EOAT, para esta intensidade, provavelmente se justifica pelo fato de a EOAT se encontrar presente em limiares de até 35 dB NA. Dessa forma, poder-se-ia sugerir que limiares auditivos até $35 \mathrm{~dB}$ NA correspondem a intensidade de ponto de corte de $50 \mathrm{~dB}$ NPS.

Estudos realizados com o exame de PEAEE, utilizando tons puros modulados como estímulo, sugerem que $50 \mathrm{~dB}$ NPS seja utilizada como intensidade de ponto de corte para definição de 'passa'/'falha' em programas de triagem auditiva neonatal5 ${ }^{5-7}$. Entretanto, Picton et $\mathrm{al}^{3} \mathrm{e}^{\mathrm{J}} \mathrm{John}$ et $\mathrm{al}^{4}$ afirmam que diferenças nos parâmetros de aquisição e avaliação, entre os estudos, dificultam a suas comparações.

Como os exames utilizados em programas de triagem auditiva neonatal devem ser, dentre outros fatores, rápidos, o tempo do exame torna-se um fator a ser analisado. No presente estudo, observouse que à medida que a intensidade do estímulo aumentou, diminuiu a quantidade do número de amostras para se obter as respostas e, conseqüentemente, diminuiu o tempo do exame (cada varredura teve uma duração de 16 segundos).

Entretanto, essa relação - aumento de intensidade, diminuição do número de varreduras e diminuição do tempo de realização do exame - contribuiu para uma diminuição na correlação de 'passa'. Mais uma vez, 50 dB NPS apresentou-se como a intensidade ideal a ser aplicada como ponto de corte, pois além do exame apresentar $100 \%$ de correlação com os resultados apresentados pelo exame de EOAT, o tempo de realização do exame foi de $5,04 \mathrm{~min}(\mathrm{DP}=4,38)$.

Luts et $\mathrm{al}^{25}$ realizou um estudo com triagem auditiva utilizando o exame de PEAEE com tom puro, em crianças entre 3 e 14 meses, com tempo de realização do exame de cerca de 10 minutos. Verificaram que o tempo de realização do exame foi maior, pois a utilização do exame de PEAEE em neonatos apresentam maior ruído de fundo no EEG ${ }^{26}$.

Picton et $\mathrm{al}^{3}$ e John et $\mathrm{al}^{4}$ afirmam que o exame de PEAEE, utilizando ruído como estímulo, em triagem auditiva neonatal, poderia ser realizado em dois terços do tempo, quando comparados a estimulação tonal, pois gera respostas de maior amplitude, sendo diferenciadas do ruído de fundo mais facilmente e, portanto, determinadas mais rapidamente.

Ainda quando se compara o tempo de realização do exame de EOAT com o tempo de realização do exame de PEAEE, observa-se que o primeiro dura entre 3 e 5 minutos ${ }^{27-29}$, ao passo que o tempo do exame do PEAEE dura cerca de 5 minutos.

Sabe-se, entretanto, que o estímulo do tipo ruído branco possui algumas limitações, entre elas, o fato de ser um estímulo que somente realiza uma varredura na cóclea e não detecta alterações em regiões específicas $^{23,30}$.

\section{CONCLUSÃO}

Os resultados desse estudo, utilizando-se o ruído branco modulado em amplitude como estímulo no exame do PEAEE, sugerem o uso da intensidade de $50 \mathrm{~dB}$ NPS para triagem auditiva neonatal. Dessa forma, limiares auditivos de até $35 \mathrm{~dB}$ NA correspondem a intensidade de ponto de corte de $50 \mathrm{~dB}$ NPS.

Apesar da utilização do exame de PEAEE ser mais bem estabelecida como ferramenta de diagnóstico, pois tem demonstrado capacidade de quantificar adequadamente perdas auditivas sensórioneurais, nos primeiros meses de vida, seu uso, com estímulo de ruído de amplitude modulada, para triagem auditiva neonatal, ainda está por ser determinado.

Outros estudos precisam ser realizados com uma população maior e com grupos que tenham diagnóstico audiológico determinado, a fim de confirmar os achados do presente estudo. 


\section{ABSTRACT}

Purpose: to check the use of amplitude modulated white noise as a stimulus for the auditory steady state response. Method: thirty neonates were evaluated with transient otoacoustic emissions and auditory steady state response, with amplitude modulate white noise, at the Clinical Hospital in the Federal University of Pernambuco. Results: for the auditory steady state response, it is suggested that the cut point intensity related with normal hearing is $50 \mathrm{~dB}$ SPL, as it was related to the presence of transient otoacoustic emissions. Conclusion: the auditory steady state response, evoked with amplitude modulate white noise seems to be promissory as a tool for hearing screening. More studies need to be done in order to better establish the hearing threshold and to define more precise protocols for its employment.

KEYWORDS: Triage; Hearing; Evoked Potentials, Auditory

\section{REFERÊNCIAS}

1. Comitê Brasileiro sobre Perdas Auditivas na Infância (CBPAI).1 $1^{\text {a }}$ Recomendação - período neonatal. Correios da SBP jan/mar. 2001; ano 7.

2. Joint Committee on Infant Hearing Year 2000 position statement: principles and guidelines for early hearing detection and intervention programs. Pediatrics 2000;106:798-817.

3. Picton TW, John MS, Dimitrijevic A, Purcell DW.(2003). Human auditory steady-state responses. InternationalJournal of Audiology, 2003;42:177-219.

4. John MS, Brown DK, Muir DJ, Picton TW. (2004). Recording auditory steady state responses in young infants. Ear Hear 2004;25: 539-53.

5. Savio G, Perez-Abalo MC.(2008). Auditory Steady-state Responses and Hearing Screening. In: Rance G , org. Auditory Steady-State Responses. Generation, Recording and Clinical Application. Plural Publishing, p. 185-200.

6. Lins OG, Picton TW. (1995). Auditory steadystate responses to multiple simultaneous stimuli. Electroenc Clinic Neurophys 1995;96:420-32.

7. Lins OG, Picton $T W$, Boucher BL, DurieuxSmith A, Champagne SC, Moran LM, Perez-Abalo MC, Martin V, Savio G. (1996). Frequency specific audiometry using steady state responses. Ear Hear 1996;17: 81-96.

8. John MS, Dimitrijevic A, Picton TW. (2003). Efficient stimuli for evoking auditory steady-state responses. Ear Hear 2003;24: 406-23.

9. Griz S, Barbosa CP, Almeida e Silva AR, Ribeiro MA, Menezes DC. (2010). Aspectos demográficos e socioeconômicos de mães atendidas em um programa de triagem auditiva neonatal. Rev. Soc. Bras. Fonoaudiol 2010;15(2):179-83.
10. Griz S, Cabral M, Azevedo G, Ventura L. (2007). Audiologic results in patients with moebius sequence. I J Pediat Otorrinolaryng 2007;71:1457-63.

11. Ciorba A, Hatzopoulos S, Camurri L, Negossi L, Rossi M, Cosso D, Petruccelli J, Martini A. (2007). Neonatal newborn hearing screening four years experience at Ferrara University Hospital. Otorhinolaryngol Ita. 2007;27(1): 10-6.

12. Garcia MV, Azevedo MF, Testa JR.(2009). Accoustic immitance measures in infants with 226 and $1000 \mathrm{~Hz}$ probes: correlation with otoacoustic emissions and otoscopy examination.Braz J Otorhinolaryngol. 2009;75(1):80-9.

13. Hatzopoulos S, Petruccelli J, Ciorba A, Martini A. (2009).Optimizing Otoacoustic emissions protocol for a UHNS program. Audiol Neurotol. 2009;14:7-16. 14. Khandekar R, Khabori M, Jaffer MA, Gupta R. (2006). Neonatal screening for hearing impairmentThe Oman experience. I J Pediat Otorhinolaryngol. 2006; 70:663-70.

15. Herglis L. (2007). Analysis of measurements of the first Swedish universal neonatal hearing screening program. I J Audiol 2007;46:680-6.

16. Olusanya B, Wirz S, Luxon L. (2008). Hospitalbased universal newborn hearing screening for early detection of permanent congenital hearing loss in Lagos, Nigeria.I J Pediatr Otorhinolaryngol. 2008;72:991-1001.

17. Santos AF, Durante AS, Almeida K, Taguchi CK, Grecco MC.(2009). Características das emissões otoacústicas em lactentes expostos à medicação ototóxica.Rev Soc Bras Fonoaudiol. 2009;14:521-7. 18. Sanford CA, Keefe DH, Liu YW, Fitzpatrick D, McCreery RW,Lewis DE, Gorga MP. (2009). Sound conduction effects on distortion product otoacoustic emission screening outcomes in newborn infants: test performance of wideband acoustic transfer functions at $1 \mathrm{KHz}$ timpanometry. Ear Hear. 2009;30: 635-52. 
19. Garcia CFD, Isaac ML, Oliveira JAA. (2002). Emissão otoacústica evocada transitória: instrumento para detecção precoce de alterações auditivas em recém-nascidos a termo e prétermo. Rev Bras Otorrinolaringol. 2002;68:344-52.

20. Pereira PKS, Martins AS, Vieira MR, Azevedo MF. (2007). Programa de triagem auditiva neonatal: associação entre perda auditiva e fatores de risco. Pró-fono Rev Atual Cient. 2007;19:267-78.

21. Cone-wesson B, Dowel RC, Tomlin D, Rance G, Ming WJ.(2002). The auditory steady-state response: comparisons with the auditory brainstem response. Am Acad Audiol. 2002;13: 173-87.

22. Rance G; Rickards F. (2002).Prediction of hearing threshold in infants using auditory steady-state evoked potentials. Am Acad Audiol. 2002;13:236-45.

23. Sávio G, Perez - Abalo MC, Gaya J, Hernandez O, Mijares E. (2006)Test Accuracy and prognostic validity of multiple auditory steady state responses for targeted hearing screening. Int J Audiol. 2006;45: 109-20.

24. Ribeiro FM, Carvallo RM, Marcoux AM.(2010). Auditory steady state evoked responses for preterm and term neonates. Audiol Neurotol. 2010;15:97-110.
25. Luts H, Desloovere C, Kumar A, Vandermeersch E, Wouters J. (2004)Objective assessment of frequency-specific hearing thresholds in babies.I J Pediatr Otorhinolaryngol. 2004;68:915-26.

26. Rance G, Tomlin D. (2006). Maturation of auditory steady-state responses. Ear Hear. 2006;27:20-9.

27. Segre AMC. (2003).Prevalência da perda auditiva em recém-nascidos de muito baixo peso. Jorn Pediat. 2003;79:103-4.

28. Martins CHF, Vassoler TMF, Bergonse GFR, Alvarenga KF, Costa OA.(2007).Emissões otoacústicas e potencial evocado auditivo de tronco encefálico em trabalhadores expostos a ruído e ao chumbo.Téc Otorrinolaring. 2007;25:293-8.

29. Barros SMS, Frota S, Atherino CCT, Osterne F. (2007). The efficiency of otoacoustic emissions and pure-tone audiometry in the detection of temporary auditory changes after exposure of high sound pressure levels. Rev Bras Otorrinolar. 2007;73:592-8.

30. Swanepoel DW, Hugo R, Hoode R. (2004). Auditory steady-state responses for children with severe to profound hearing loss. Otolaryngol head neck surg. 2004;130: 531-5.
http://dx.doi.org/10.1590/S1516-18462011005000038

RECEBIDO EM: 28/10/2010

ACEITO EM: 12/01/2011

Endereço para correspondência:

Danielle Gomes Pinto

Rua Bandeira Filho, n. 155

Graças - Recife - PE

CEP: $52020-210$

E-mail: danigp84@yahoo.com.br 